\title{
Adipose tissue metabolism and inflammation are differently affected by weight loss in obese mice due to either a high-fat diet restriction or change to a low-fat diet
}

\author{
Femke P. M. Hoevenaars $\cdot$ Jaap Keijer • \\ Laure Herreman • Inge Palm • Maria A. Hegeman • \\ Hans J. M. Swarts • Evert M. van Schothorst
}

Received: 7 November 2013/Accepted: 20 February 2014/Published online: 10 April 2014

(c) Springer-Verlag Berlin Heidelberg 2014

\begin{abstract}
Restriction of a high-fat diet (HFD) and a change to a low-fat diet (LFD) are two interventions that were shown to promote weight loss and improve parameters of metabolic health in obesity. Examination of the biochemical and molecular responses of white adipose tissue (WAT) to these interventions has not been performed so far. Here, male C57BL/6JOlaHsd mice, harboring an intact nicotinamide nucleotide transhydrogenase gene, were fed a purified 40 energy $\%$ HFD for 14 weeks to induce obesity. Afterward, mice were divided into three dietary groups: HFD (maintained on HFD), LFD (changed to LFD with identical ingredients), and HFD-CR (restricted to $70 \%$ of the HFD). The effects of the interventions were examined after 5 weeks. Beneficial effects were seen for both HFD-CR and LFD (compared to HFD) regarding physiological parameters (body weight and fat mass) and metabolic parameters, including circulating insulin and leptin levels. Macrophage infiltration in WAT was reduced by both interventions, although more effectively by HFDCR. Strikingly, molecular parameters in WAT differed between HFD-CR and LFD, with increased activation of mitochondrial carbohydrate and fat metabolism in HFDCR mice. Our results confirm that restriction of the amount of dietary intake and reduction in the dietary energy
\end{abstract}

Electronic supplementary material The online version of this article (doi:10.1007/s12263-014-0391-9) contains supplementary material, which is available to authorized users.

F. P. M. Hoevenaars - J. Keijer - L. Herreman - I. Palm · M. A. Hegeman - H. J. M. Swarts - E. M. van Schothorst ( $)$ Human and Animal Physiology, Wageningen University, De Elst 1, PO box 338, 6700 AH Wageningen, The Netherlands

e-mail: Evert.vanschothorst@wur.nl

F. P. M. Hoevenaars

e-mail: femke.hoevenaars@wur.nl content are both effective in inducing weight loss. The larger decrease in WAT inflammation and increase in mitochondrial carbohydrate metabolism may be due to a larger degree of energy restriction in HFD-CR, but could also be due to superior effectiveness of dietary restriction in weight loss strategies.

Keywords Caloric restriction - Fat restriction - Nutrition . Metabolic health - White adipose tissue

\section{Introduction}

Obesity would be less of a problem if it was not a major risk factor for non-communicable diseases such as cardiovascular diseases, type 2 diabetes, and some sorts of cancer (Burton et al. 1985). Obesity is a condition of superfluous white adipose tissue (WAT) mass. Next to its function as an energy reservoir, WAT is recognized as an endocrine organ responsible for secretion of adipocytokines (Kershaw and Flier 2004; Wang et al. 2008). Changes in this secretory function of adipose tissue have been associated with obesity and with dysfunction of the adipose tissue. Adipose tissue dysfunction may result in ectopic lipid deposition which is detrimental for normal tissues (Zhou and Grill 1994). Together with a state of chronic low-grade inflammation, an increased risk of development of non-communicable diseases is created.

To improve or even restore health in an obese condition, a negative energy balance is needed, leading to fat mass reduction and thus to weight loss. Indeed, a small weight reduction of $5 \%$ already leads to improved health outcomes (Goldstein 1992). This can be achieved by either restriction of calorie intake maintaining the same dietary composition, or by a reduction in fat intake, that is, by 
changing the macronutrient composition of the consumed diet. Both have been shown to be successful in humans: Caloric restriction (CR) improves glycemic control (Heilbronn et al. 1999), and reduction in fat intake, without intentional restriction of energy intake, induces weight loss (Astrup et al. 1997). The optimal macronutrient composition of weight reducing diets is still under debate.

When allowed ad libitum access to a high-fat diet (HFD), C57BL/6J mice develop insulin resistance and obesity in resemblance to disease progression in humans (Surwit et al. 1988). Like in humans, weight loss in diet-induced obese rodents can be obtained with various diet intervention strategies. However, only few studies investigated this from a nutritional point of view. Reduction in body weight, fat mass, and several metabolic parameters was obtained using a standard percentage of CR, a $30 \%$ reduction, of a HFD (Kalupahana et al. 2011), without changing the macronutrient composition. A substantial improvement of an obese condition, reduction in body weight and improvement of insulin sensitivity, was also obtained by a reduction in the fat percentage in the diet (i.e., by a change from a HFD to a LFD) (Muurling et al. 2002). These studies showed that dietary energy restriction and dietary fat reduction are both effective in weight reduction in diet-induced obesity (DIO). However, a number of questions remain. In particular, it is not known how these interventions impact adipose tissue at the functional and molecular level and to which extent this differs between these intervention strategies. This information is important in the development of dietary antiobesity interventions.

The aim of this study is to compare the effects on adipose tissue of two different body weight loss strategies: a standard 30 energy\% (en\%) CR (i.e., energy restriction of HFD) and a change from ad libitum HFD to ad libitum LFD (i.e., restriction of fat intake). These interventions were done using purified diets containing the same ingredients, with only the amounts of carbohydrate and fat differing between the diets. C57BL/6JOlaHsd mice were used. Similar to other C57BL/6J sub-strains, C57BL/6JOlaHsd is sensitive to DIO (Surwit et al. 1988). This substrain has an intact nicotinamide nucleotide transhydrogenase (Nnt) gene, which is in contrast to most C57BL/6J sub-strains that were used in other studies investigating weight loss by HFD restriction or a change to LFD. Intact and functional NNT was recently shown to be important for cellular redox control (Ronchi et al. 2013).

In this study, the interventions started from an obesogenic condition, that is, after 14 weeks of HFD feeding. The effects of both interventions were examined after 5 weeks, which we expected to be at the end of the adaptation period based on previous studies (Hoevenaars et al. 2013a).

We report here that a 30 en\% restriction of a HFD induces more pronounced effects than a change to LFD, in particular, regarding WAT inflammation and expression of mitochondrial carbohydrate metabolism genes.

\section{Materials and methods}

Animal study and diets

Male wild-type C57BL/6JOlaHsd mice were purchased from Harlan (Horst, The Netherlands) at 8 weeks of age and were housed individually in a controlled environment (12 h light/ dark cycle, $55 \%$ humidity, temperature at $22{ }^{\circ} \mathrm{C}$ ). Access to water and food (Research Diet Services BV, Wijk bij Duurstede, The Netherlands) was ad libitum and renewed every week. Mice were acclimatized to the housing conditions for four weeks on a low-fat $10 \mathrm{en} \%$ purified diet (LFD; Supplemental table 1). During a 14 week run-in, mice were given a 40 en\% high-fat purified diet (HFD; Supplemental table 1) to induce obesity. After the run-in, 36 mice were stratified on body weight into 3 groups: HFD continued on HFD, LFD was changed to the purified LFD, and HFD-CR received $70 \%$ of the individual HFD consumption-as measured over weeks 10-12 during the run-in —on a daily basis $2 \mathrm{~h}$ before the start of the dark phase. Indirect calorimetry was performed for HFD and HFD-CR mice after 4 weeks of intervention as published (Hoevenaars et al. 2013a). After 5 weeks of intervention at the age of 31 weeks, all mice were killed. All analyses are based on 12 mice per dietary group, except for histological analysis $(n=5-6)$ and indirect calorimetry $(n=3-6)$. Body weight and food intake were monitored weekly. The experimental protocol was approved by the animal welfare committee of Wageningen University, Wageningen, The Netherlands.

\section{Tissue collection}

Food was removed $2 \mathrm{~h}$ before mice were killed in the morning. Mice were anesthetized by $5 \%$ isoflurane inhalation. Blood was collected in Mini collect serum tubes (Greiner Bio-one, Longwood, FL) via orbital exsanguination and processed as published (Hoevenaars et al. 2013a). After blood collection, mice were killed by cervical dislocation. The left portion of epididymal and peri-renal WAT (eWAT and pWAT, respectively) were isolated, weighed and snap frozen in liquid nitrogen, and stored at $-80{ }^{\circ} \mathrm{C}$. The right-sided portions of eWAT were fixed overnight in $4 \%$ paraformaldehyde, washed with $70 \%$ ethanol, and subsequently embedded in paraffin.

Adipose tissue morphology

Paraffin-embedded eWAT was sectioned at $5 \mu \mathrm{m}$ and immunohistochemically stained to measure mean adipocyte area as published (Hoevenaars et al. 2013b). Macrophage 
infiltration was determined as a marker for tissue inflammation. Briefly, eWAT sections were deparaffinized and incubated with a monoclonal antibody against the macrophage marker MAC-2 (Cedarlane Laboratories Limited, Burlington, Ontario, Canada), using chemicals from Vector laboratories, Burlingame, California, United states, unless stated otherwise. Briefly, endogenous peroxidase was inactivated by incubation for $30 \mathrm{~min}$ in $1 \% \mathrm{H}_{2} \mathrm{O}_{2}$ in methanol. Sections were rinsed with PBS after which they were blocked with $5 \%$ normal goat serum in PBS-BSAc (Aurion, Wageningen, The Netherlands). Sections were incubated overnight at $4{ }^{\circ} \mathrm{C}$ with a monoclonal anti-MAC2 antibody (diluted 1:5000 in PBS-BSAc). Next, sections were rinsed in PBS and incubated for $60 \mathrm{~min}$ at room temperature (RT) with a secondary goat anti-rat biotinylated antibody diluted 1:200 in PBS-BSAc, rinsed again, and incubated for $60 \mathrm{~min}$ at RT with Vectastain Elite $\mathrm{ABC}$ reagent (dilution 1:1000). After rinsing, the sections were incubated for $2 \mathrm{~min}$ at RT with 3,3'-diaminobenzidine solution (dilution 1:200), rinsed, counter stained with hematoxylin QS, and mounted with VectaMount. Cells were considered to stain positively for MAC-2 when a brown substrate was present in the cytoplasm of the cells. Tissues were examined under an Axioskop 2 light microscope (Zeiss, Göttingen, Germany), and digital images were taken using an Axiocam MRC 5 camera (Zeiss). The number of crown-like structures (CLS) was counted per 1,000 adipocytes per mouse, and macrophage infiltration was expressed as a total number of CLS per 100 adipocytes.

\section{Serum measurements}

Serum levels of leptin and insulin were measured using the mouse serum adipokine milliplex multianalyte kit (Millipore Corporation, Billerica, MA) according to the manufacturers' protocol using the Bio-Plex 200 system (Biorad, Veenendaal, The Netherlands). Serum glucose was determined by the GOD-PAP colorimetric assay (Roche diagnostics, Woerden, The Netherlands). Serum free fatty acids (FFA) levels were measured using the NEFA-C kit (Wako chemicals, GmbH, Neuss, Germany). Volumes were scaled down to analyze samples with a microplate reader (BioTec Synergy HT, Bad Friedrichshall, Germany). All measurements were performed in duplicate and averaged. Homeostatic assessment of insulin resistance (HOMA2-IR) was calculated using fasting glucose and insulin levels as published using the excel program [http://www.dtu.ox.ac. uk/homacalculator/index.php; (Levy et al. 1998)].

RNA/DNA isolation and cDNA synthesis

RNA isolation was performed as published (Van Schothorst et al. 2005). All samples were purified with RNeasy columns (Qiagen) according to the manufacturers' instructions.

Total DNA was extracted from homogenized eWAT by digestion with proteinase $\mathrm{K}$ in a lysis buffer $(50 \mathrm{mM}$ TrisHCL, pH 7.5, 0.5\% SDS and 12.5 mM EDTA, $\mathrm{pH}$ 8.0). Homogenate samples were incubated overnight at $56^{\circ} \mathrm{C}$. Proteinase $\mathrm{K}$ was inactivated by a 10 -min incubation at $70{ }^{\circ} \mathrm{C}$. Samples were then incubated with $40 \mu \mathrm{g} / \mathrm{ml}$ RNAse A (Sigma-Aldrich, St. Louis, USA) for $1 \mathrm{~h}$ at $37{ }^{\circ} \mathrm{C}$. After centrifugation, the aqueous phase was mixed and extracted with an equal amount of phenol-chloroform-isoamylacohol and twice with chloroform. DNA was precipitated by $96 \%$ ethanol and sodium acetate (3 M, pH 5.2). DNA was washed with $750 \mu \mathrm{l}$ of cold $70 \%$ ethanol, air-dried, and resuspended in $30 \mu \mathrm{l}$ of RNAse/DNAse-free water. RNA/ DNA concentration and purity were assessed with the Nanodrop spectrophotometer (IsoGen Life Science, Maarsen, The Netherlands). RNA integrity was checked by capillary zone electrophoresis (Experion, Bio-Rad). DNA concentration was adjusted to $40 \mathrm{ng} / \mu \mathrm{l}$ with RNAse/ DNAse-free water. RNA of all individual samples was reversely transcribed using the iScript cDNA synthesis kit (Bio-Rad).

Gene expression by quantitative RT-PCR (qRT-PCR)

Differential transcript expression was assessed by qRT-PCR using iQ SYBR Green Supermix (Bio-rad) and the MyIQ single-color real-time PCR detection system (Bio-rad). A standard curve for all transcripts including reference transcripts was made using serial dilutions of a pool prepared from all cDNA samples. Relative levels of gene expression were obtained using two reference genes (Ribosomal protein s15 (Rps 15) and Calnexin (Canx)) in duplicate and averaged. Target genes, primer sequences, and annealing temperatures are shown in Table 1. The expression of the gene of interest was normalized against the geometrical mean of the reference genes Rps15 and Canx which were chosen based on stable gene expression levels (geNorm, Ghent University Hospital, Ghent, Belgium).

\section{Mitochondrial density}

Relative mitochondrial density was determined as the ratio of mitochondrial DNA to nuclear DNA, as published (Lagouge et al. 2006). The mean of the control group (HFD) was set as 1.0. The primer pair's sequences used were as follows: mitochondrial DNA (forward) $5^{\prime}$-CCGCA AGGGAAAGATGAAAGAC- $3^{\prime}$ and (reverse) $5^{\prime}$-TCGTTT GGTTTCGGGGTTTC-3' (Lagouge et al. 2006); nucleic DNA (forward) 5'-CTTAGAGGGACAAGTGGCGTTC and (reverse) 5'-CGCTGAGCCAGTCAGTGTAG-3' (Park et al. 2009). 
Table 1 Primer sequences and annealing temperature of primers used for quantitative RT-PCR
Genes denoted with an asterisk were used as reference genes

\begin{tabular}{llll}
\hline Genes & Forward primer $5^{\prime}-3^{\prime}$ & Reverse primer $5^{\prime}-3^{\prime}$ & $T\left({ }^{\circ} \mathrm{C}\right)$ \\
\hline Canx* & GCAGCGACCTATGATTGACAACC & GCTCCAAACCAATAGCACTGAAAGG & 58.5 \\
Cpt1a & AAAGATCAATCGGACCCTAGACA & CAGCGAGTAGCGCATAGTCA & 60.0 \\
Cpt1b & ACCCCTAAGGATGCCATTCTTG & GCGGAAGCACACCAGGCAGTA & 57.0 \\
Esrra & ATCCAGGGGAGCATCGAGTA & AAAGGCAAAGGGTCCACCTC & 60.0 \\
Fasn & AGTTAGAGCAGGACAAGCCCAAG & TTCAGTGAGGCGTAGTAGACAGTG & 60.0 \\
Mpc1 & GACTTTCGCCCTCTGTTGCT & GCAGATGGCCGCTTACTCAT & 58.5 \\
Mpc2 & ACCTACCACCGACTCATGGA & AGTTTCTCTGCAGGTCTGGC & 60.0 \\
Lipe & TGGAACTAAGTGGACGCAAGCC & TCAAGGTATCTGTGCCCAGTAAGC & 58.0 \\
Pdha1 & CTGCCTATTGCAGGTCTGGT & CTTCTCGAGTGCGGTAGCTT & 60.0 \\
Pdhb & GAAAGGCAAGGGACCCACAT & CCTCCTTCCACAGTCACGAG & 60.0 \\
Pdk1 & GCTACTCAACCAGCACTCCTT & GGTCGCTCTCATGGCATTCT & 60.0 \\
Pnpla2 & ACCACCCTTTCCAACATGCTACC & GCTACCCGTCTGCTCTTTCATCC & 58.0 \\
Ppargc1a & CCCTGCCATTGTTAAGACC & TGCTGCTGTTCCTGTTTC & 60.0 \\
Rps15* & CGGAGATGGTGGGTAGCATGG & ACGGGTTTGTAGGTGATGGAGAAC & 58.5 \\
S100a8 & ACTTCGAGGAGTTCCTTGCG & TGCTACTCCTTGTGGCTGTC & 58.0 \\
\hline
\end{tabular}

Citrate synthase activity

Snap-frozen eWAT was homogenized in liquid nitrogen and diluted to a fixed protein concentration of $0.8 \mu \mathrm{g} / \mu \mathrm{l}$ in PBS-0.5\% Triton X-100 buffer containing $1 \%$ protease inhibitor cocktail. Citrate synthase (CS) activity was measured spectrophotometrically using the CS Assay Kit (Sigma-Aldrich) according to the manufacturers' protocol. Briefly, eWAT homogenates were transferred to $10 \mathrm{mM}$ DNTB (5,5'-dithiobis-(2-nitrobenzoic acid)) and $30 \mathrm{mM}$ acetyl-CoA. CS activities were measured for $60 \mathrm{~min}$ at $412 \mathrm{~nm}$ at $25{ }^{\circ} \mathrm{C}$ after addition of $10 \mathrm{mM}$ oxaloacetate. CS activities were then calculated using the slope of the linear increase according to the manufacturer's protocol. The mean of the control group (HFD) was set as 1.0.

\section{Liver triglycerides}

Triglyceride kit reagent (triglycerides Liquicolor kit, Human, Wiesbaden, Germany) was used according to protocol as described (Hoek-van den Hil et al. 2012). Snapfrozen liver was homogenized and dissolved in a $10 \mathrm{mM}$ Tris, $2 \mathrm{mM}$ EDTA, and 0.25 M sucrose buffer with $\mathrm{pH}$ 7.5. Input was corrected for wet tissue weight.

Statistical analysis

Data are expressed as mean \pm SEM; statistical analyses were performed with GraphPad Prism 5.04 (Graphpad software, San Diego, CA, USA). All measurements within the different treatment groups were checked for normality with D'Agostino \& Pearson normality test. qRT-PCR data was $\log 2$-transformed before statistical analysis. Normal distributed data were analyzed by one-way analysis of variance and Tukey's multiple comparison post hoc analysis. Test results of serum FFA and gene expression data of Esrra, Ppargcla, Mpc1, Mpc2, Pdha1, Pdhb, Pdk1, and Fasn were not normally distributed and therefore analyzed by nonparametric Kruskal-Wallis test and Dunn's multiple comparison post hoc test. $P<0.05$ was considered significant.

\section{Results}

LFD and HFD-CR decreased body weight and adiposity

Body weight of DIO mice declined immediately after the change from HFD to either HFD restriction (HFD-CR) or LFD. Mean body weight stabilized after 3 weeks in LFD mice, while it further declined till the end of the 5-week intervention period in HFD-CR mice (Fig. 1a). There were no differences in eWAT and pWAT weights between LFD and HFD-CR mice, but both were significantly lower than control HFD mice (Fig. 1b, c). In contrast, weight gain/loss was significantly different between all group comparisons; HFD gained $4.2 \pm 0.5 \mathrm{~g}$, while LFD and HFD-CR lost $4.1 \pm 0.4 \mathrm{~g}$ and $9.0 \pm 0.5 \mathrm{~g}(p<0.0001)$, respectively, due to the intervention. Cumulative food intake (Kcal) during the 5-week intervention was also different between all group comparisons (Fig. 2). Although mean dietary energy intake level was the lowest in HFD-CR, the contribution of lipids to total energy availability from the diet remained clearly higher for HFD-CR mice in comparison with LFD mice (Fig. 2). Indirect calorimetry revealed a clear cyclic pattern in respiratory exchange ratio (RER) for 


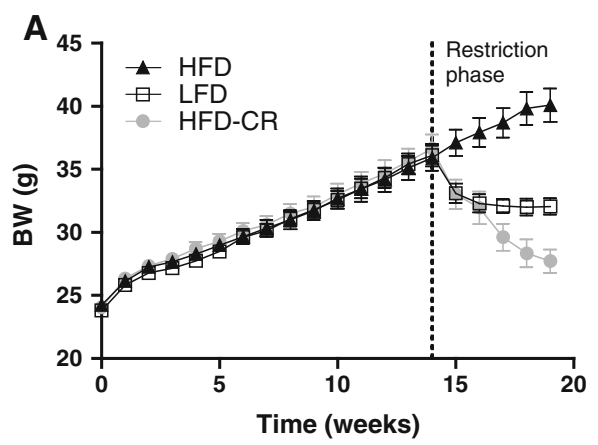

Fig. 1 Changes in body weight (a) and adipose tissue mass of 14-week DIO mice, followed by a 5-week diet intervention of ad libitum high-fat diet (40 en\% fat, HFD), ad libitum low-fat diet (10 en $\%$ fat, LFD), or restricted high-fat diet (30\% restriction of HFD,
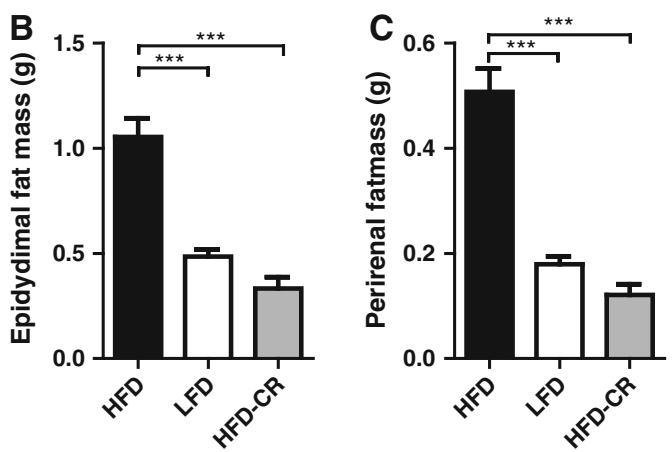

HFD-CR). b, c Represent white adipose tissue mass of epididymal (b) and peri-renal (c) origin immediately post mortem. Data are mean $\pm \operatorname{SEM}(n=12)$. *** $p<0.001$
Fig. 2 Cumulative energy intake (a) and percentage of energy intake from macronutrients (b fat, carbohydrate $(\mathrm{CHO})$, protein) during 5-week intervention. Numbers in bars represent corresponding energetic value (Kcal) of various macronutrients. Data are mean $\pm \operatorname{SEM}(n=12)$. $* * * p<0.001$

\section{A}

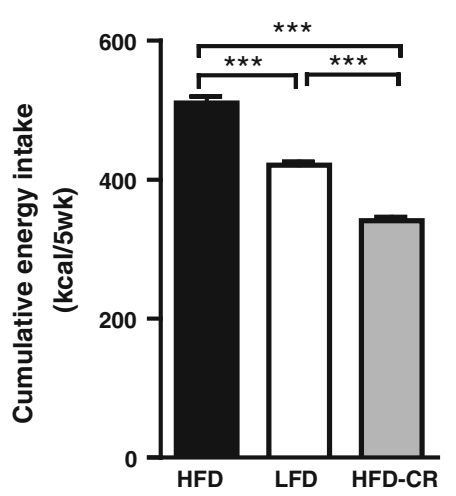

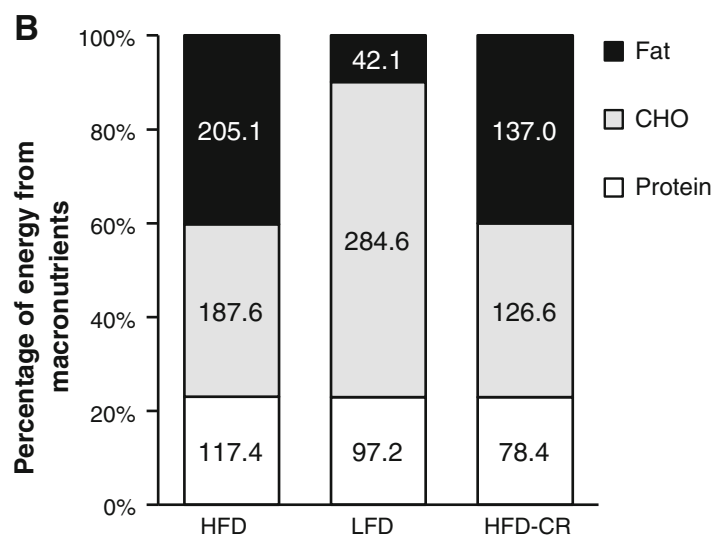

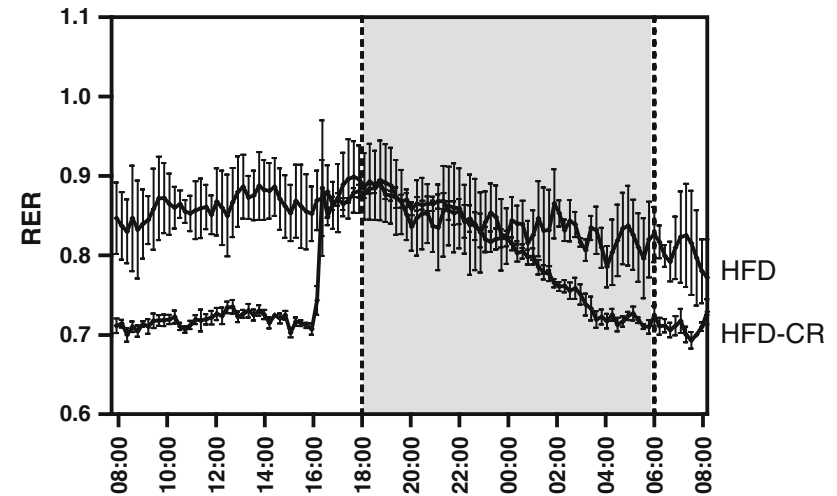

Fig. 3 Respiratory exchange ratio (RER) of 14-week DIO mice, followed by a 5-week continuation of ad libitum high-fat diet (40 en\% fat, HFD), or a 5-week restricted high-fat diet (30\% restriction of HFD, HFD-CR). Grey area represents the dark phase. Data are mean $\pm \operatorname{SEM}(n=3-6)$

HFD-CR mice. RER is higher when mice are fed (indicative for glycolysis and lipogenesis), and RER is reduced when mice are fasted (indicative for fat oxidation). In comparison, mice fed HFD ad libitum show a dampened diurnal rhythm (Fig. 3).
Adipocyte area and number of macrophages were decreased

To quantify the effects of the dietary interventions on the morphology and inflammatory status of eWAT, adipocyte area and macrophage infiltration were investigated in accordance with weight loss. Adipocyte area was significantly reduced in LFD and HFD-CR mice in a similar manner (Fig. 4a). Strikingly, inflammatory status of eWAT was considerably improved by a reduction in CLS by LFD, with an even further significant reduction by HFD-CR (Fig. 4b). Representative pictures of these CLS are shown in Fig. 4c.

\section{Serum parameters}

Health status was also determined by measuring serum levels of insulin, glucose, leptin, and FFA. Serum insulin levels were significantly lowered in LFD and HFD-CR (Fig. 5a). Glucose levels were significantly lower for HFDCR (Fig. 5b). The calculated HOMA2-IR insulin resistance index showed a similar significant reduction for LFD and HFD-CR (Fig. 5c), indicating a similar improvement in glucose homeostasis for the two dietary interventions. 
Fig. 4 Adipose tissue morphology. a Mean adipocyte area after a 5-week diet intervention of high-fat ad libitum diet (40 en\% fat, HFD), low-fat ad libitum (10 en\% fat, LFD), or high-fat restriction $(30 \%$ restriction of HFD, HFD-CR). b Observed number of crown-like structures (CLS) expressed per 100 adipocytes, as a measure for macrophage infiltration. c Representative pictures of MAC-2-stained white adipose tissue for HFD, LFD, and HFDCR. Data are mean \pm SEM $(n=5-6)$. * $p<0.05$, $* * * p<0.001$
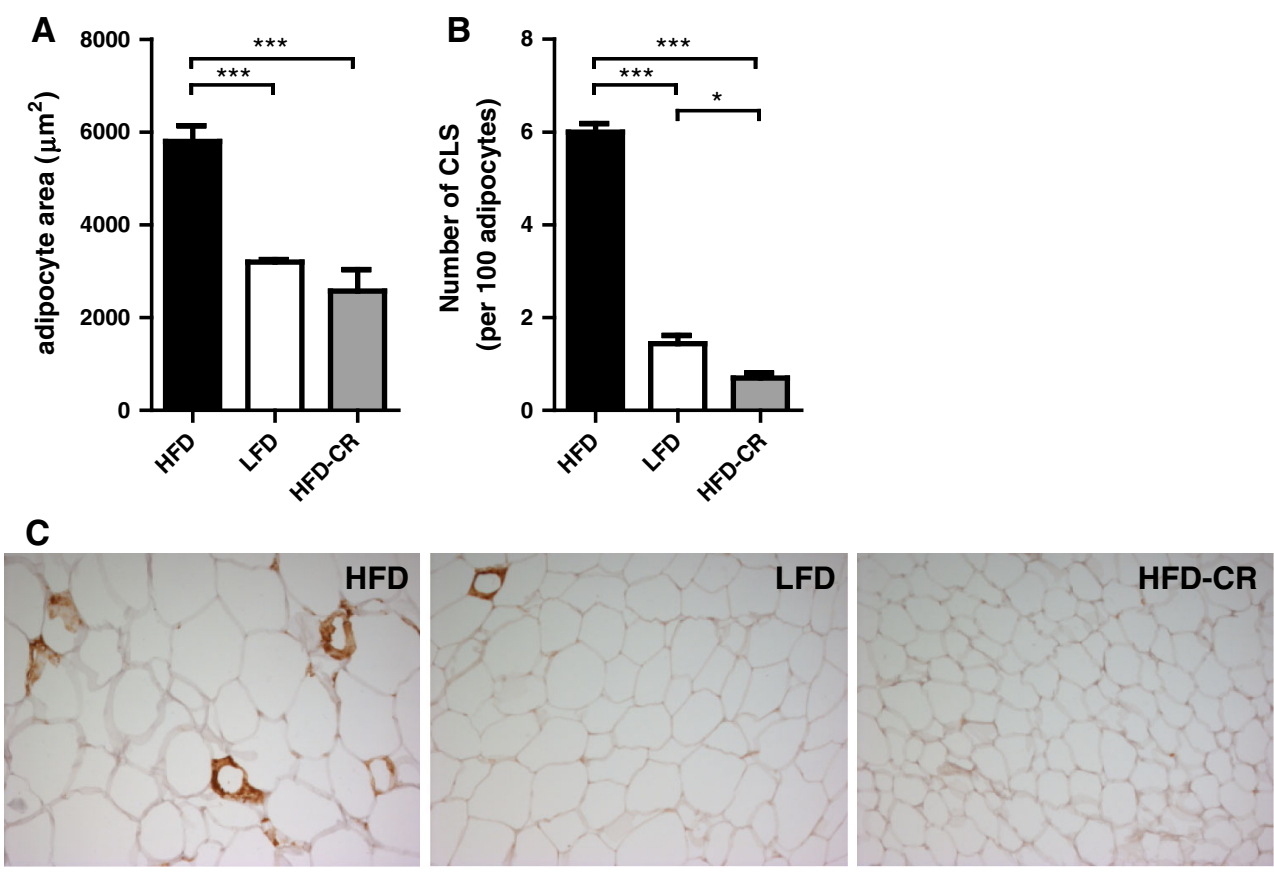

Fig. 5 Serum and tissue parameters. a Insulin levels, b glucose levels, c calculated HOMA2-IR insulin resistance index, $\mathbf{d}$ leptin levels, and $\mathbf{e}$ free fatty acids (FFA) levels in serum, and $\mathbf{f}$ triglyceride levels in liver of mice after a 5-week diet intervention of high-fat ad libitum diet (40 en\% fat, HFD), low-fat ad libitum (10 en\% fat, LFD), or high-fat restriction $(30 \%$ restriction of HFD, HFD-CR). Data are mean $\pm \operatorname{SEM}(n=12$, except for liver triglycerides $n=8$ ). $* p<0.05, * * * p<0.001$

A

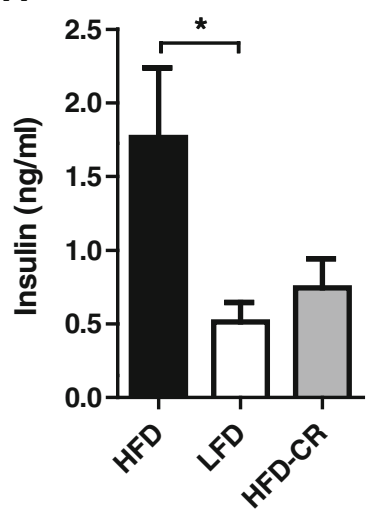

D

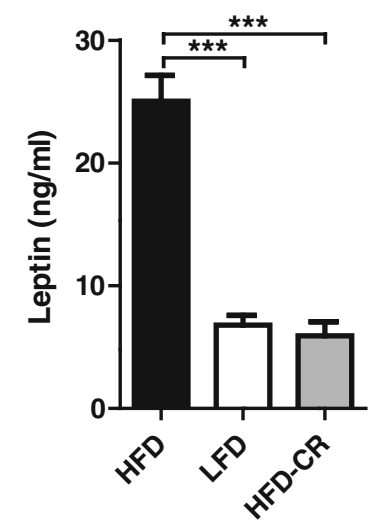

B

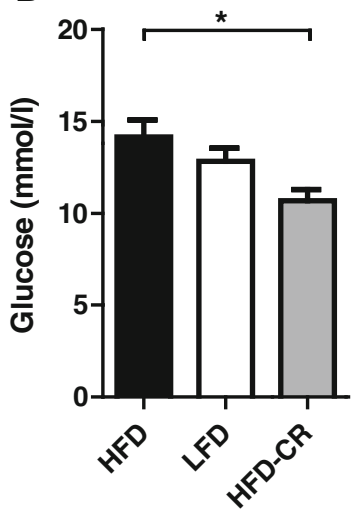

E

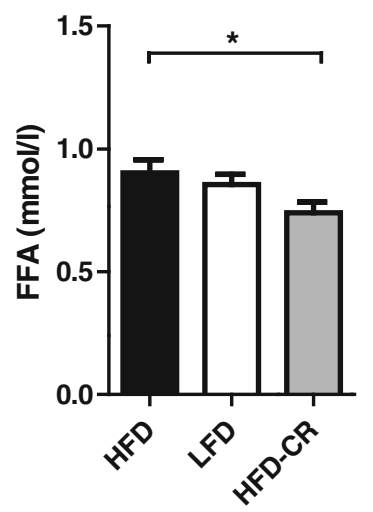

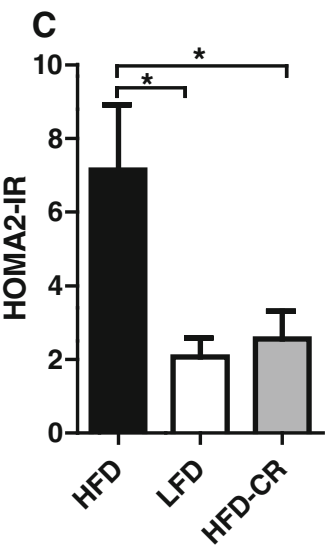

$\mathbf{F}$

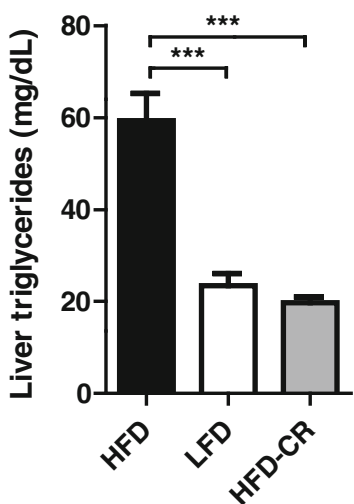

Moreover, a significant reduction was observed for serum leptin levels and FFA (Fig. 5d, e), suggesting improvement in lipid homeostasis as well. This is also shown in liver where the amount of liver triglycerides is reduced in both groups (Fig. 5f).
Mitochondrial density was not affected by both interventions

Caloric restriction of HFD or a switch to LFD did, unexpectedly, not affect eWAT mitochondrial density (Fig. 6a). 
A
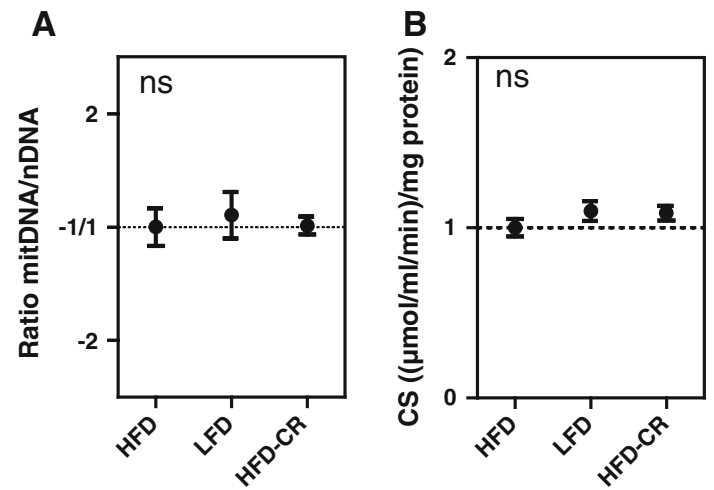

Fig. 6 Mitochondrial density in eWAT after a 5-week diet intervention of high-fat ad libitum diet (40 en\% fat, HFD), low-fat ad libitum (10 en\% fat, LFD), or high-fat restriction (30\% restriction of HFD, HFD-CR) as measured by the mitochondrial DNA copy number versus the nuclear DNA by q-PCR and citrate synthase (CS) activity assay. Data are mean $\pm \operatorname{SEM}(n=10)$. Ratio of mtDNA/nDNA and CS activity levels of HFD mice was set to 1.0

This was also reflected by equal levels of eWAT CS activity (Fig. 6b).

Gene expression in eWAT was altered mainly by HFD$\mathrm{CR}$

To investigate the molecular consequences of the dietary interventions for metabolic functions in WAT, we selected target genes for metabolism-related pathways: mitochondrial biogenesis, carbohydrate metabolism, fat metabolism, and inflammation. HFD-CR has been reported to increase mitochondrial metabolism (Duivenvoorde et al. 2011; Nisoli et al. 2005) which is induced by estrogen-related receptor alpha (ESRRA), which is linked to oxidative metabolism, and peroxisome proliferator activated receptor gamma co-activator 1 alpha (PPARGC1A), which is a master regulator of mitochondrial biogenesis (Scarpulla 2008). We observed increased expression of Ppargcl $\alpha$ for HFD-CR and LFD (compared to HFD) with the largest increase in HFD-CR. In contrast, Esrra expression levels were not significantly changed relative to the HFD control, although HFD-CR showed significantly higher expression relative to LFD (Fig. 7a). Pyruvate is the end product of glycolysis and a major substrate for the tricarboxylic acid (TCA) cycle in mitochondria, which leads to ATP formation, fatty acid synthesis, or via malate to glyceroneogenesis. Recently, the genes and transcripts for the heterocomplex of mitochondrial pyruvate carrier and importer (MPC) have been identified (Herzig et al. 2012). We analyzed the nutritional transcript regulation for both isoforms $\mathrm{Mpcl}$ and $\mathrm{Mpc2}$. Here, only a significant upregulation of $\mathrm{Mpcl}$ was found as a result of HFD-CR (Fig. 7b). Mpc2 expression levels were not significantly changed relative to the HFD control, although HFD-CR showed a higher expression relative to LFD (Fig. 7b). After import into mitochondria, pyruvate needs to be converted into acetyl-CoA for use in the TCA cycle. Pyruvate dehydrogenase E1 alpha 1 (Pdha-1) and pyruvate dehydrogenase (lipoamide) beta $(P d h b)$ catalyze this reaction, and we observed an up-regulation of both Pdha- 1 and $P d h b$ in HFD-CR but not in LFD mice, compared to the HFD control. Previous studies in our laboratory have shown a strong up-regulation of pyruvate dehydrogenase kinase, isoenzyme $1(P d k l)$ as a result of long-term HFD-CR (Duivenvoorde et al. 2011). In support, 5 weeks of HFDCR also showed a significant up-regulation of $P d k l$, but not in LFD (Fig. 7b). Next to carbohydrate metabolism, we investigated crucial transcripts of fat metabolism. As a measure of fat synthesis, we measured fatty acid synthase (Fasn), which showed an up-regulation upon HFD-CR only. We found no effects by either dietary intervention on mitochondrial import of fatty acids preceding mitochondrial fatty acid oxidation, assessed by carnitine palmitoyl transferase 1 alpha and beta (Cptla and Cpt1b, respectively) gene expression levels (Fig. 7c).

In adipose tissue, the rate limiting enzyme for lipolysis is encoded by adipose triglyceride lipase officially named patatin-like phospholipase domain containing 2 (Atgl/ Pnpla2). Its expression is regulated by nutritional status and is associated with rates of adipose tissue lipolysis. In contrast, levels of hormone-sensitive lipase (Hsl/Lipe) are not regulated by nutritional status and are decreased in acute fasting and increase after prolonged restriction (Zechner et al. 2009). Here, we show that levels of lipolysis are not affected by the restriction, although there seems to be a tendency for increased levels in the HFD-CR mice as compared to the LFD mice (Fig. 7d).

To investigate inflammatory status of the adipose tissue, S100 calcium-binding protein a8 (S100a8) transcript levels were investigated. We show a reduction in both weight loss strategies LFD and HFD-CR (Fig. 7).

\section{Discussion}

In this study, a 14-week period of high-fat feeding was used to induce DIO in male C57BL/6JOlaHsd mice. From this starting point, we intervened with a standard percentage of restriction $(30 \%)$ of intake of a purified high-fat diet (40 en $\%$ fat, HFD-CR) or with a change to a purified low-fat diet (10 en\% fat, LFD). These interventions strategies were applied for 5 weeks to mediate weight loss and improve health. Our findings confirmed the beneficial effects of both strategies, HFD-CR and LFD, with regard to physiological (body weight and adiposity) and metabolic parameters (insulin, HOMA2-IR, leptin, and FFA). Macrophage infiltration in eWAT was reduced by both interventions. The 
Fig. 7 Gene expression profiling after a 5 -week diet intervention of high-fat ad libitum diet (40 en\% fat, HFD), low-fat ad libitum (10 en\% fat, LFD), or high-fat restriction (30\% restriction of HFD, HFD-CR) as measured by qRT-PCR on selected target genes. a Mitochondrial biogenesis (Esrra, Ppargcla), b mitochondrial carbohydrate metabolism ( $\mathrm{Mpcl}, \mathrm{Mpc2}$, $P d h a 1, P d h b$, and $P d k 1), \mathbf{c}$ fatty acid metabolism (Cptla, Cptlb, and Fasn), d lipolysis (Lipe and Pnpla2), e and inflammation (S100a8)
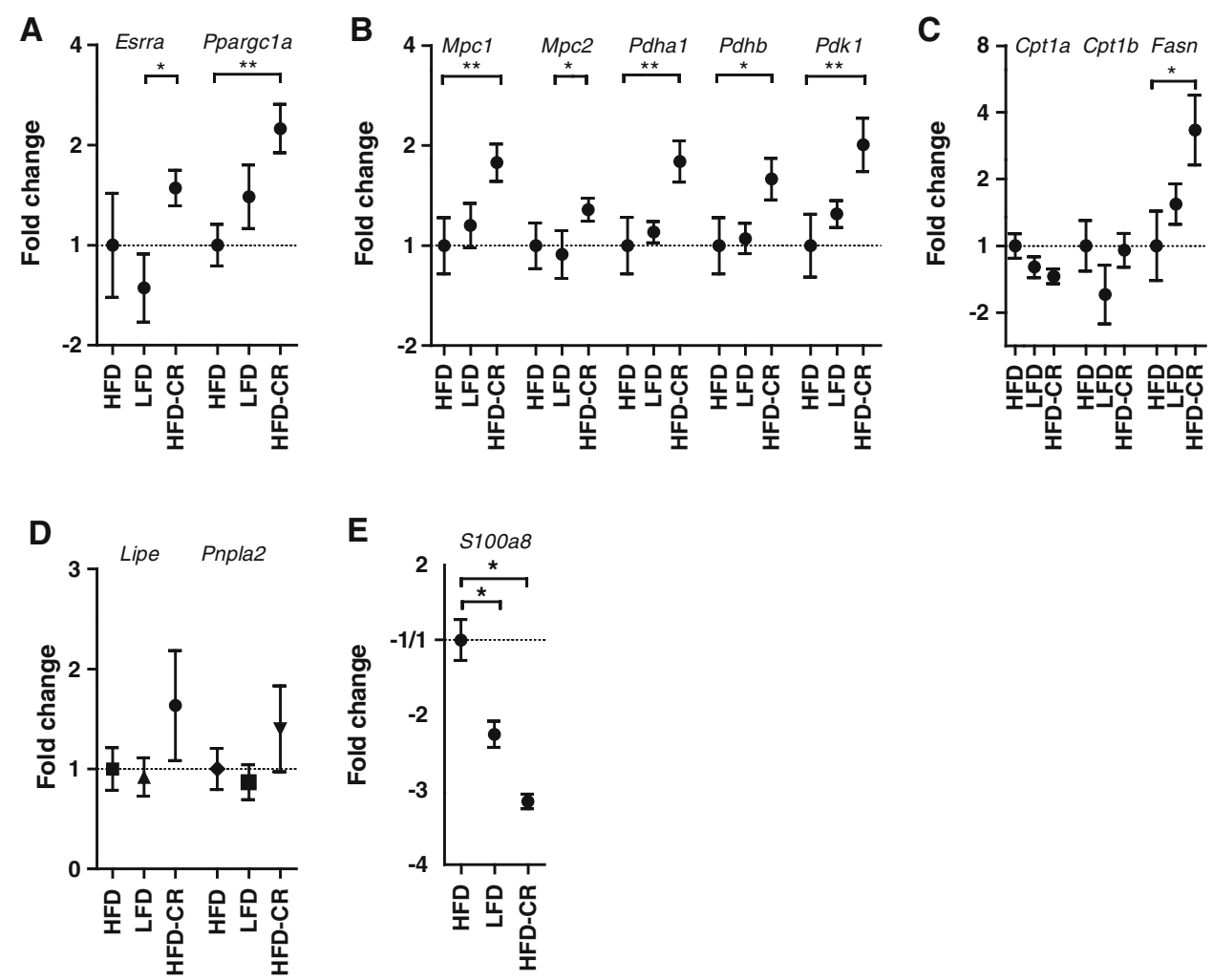

observed difference is in line with the difference that is seen between genetically obese and lean mice (Cinti et al. 2005). Remarkably, the decrease by HFD-CR was more pronounced, whereas the reduction in adipocyte size is comparable to the LFD. Similarly, eWAT expression of, in particular, mitochondrial carbohydrate metabolism genes was more affected by HFD-CR than by LFD.

Recent studies have shown that adipose tissue inflammation plays an important role in the pathogenesis of obesity as reviewed by Goossens (Goossens 2008). As there was no statistical difference in fat mass, mitochondrial density, and insulin resistance between HFD-CR and LFD mice, we expected to find no differences in eWAT infiltration by macrophages. However, we noticed that there was a higher reduction in CLS by HFD-CR compared to LFD which corresponded with reduced transcript levels of s100a8. This confirms the findings of Wang et al. (2011) who showed a reduction by HFD-CR of chemokines CCL2/monocyte chemoattractant protein-1 $(M C P-1)$ and CXCL2/macrophage inflammatory protein-2 (MIP-2) mRNA expression levels (which act on monocytes and neutrophil granulocytes, respectively), and $\mathrm{CD} 11 \mathrm{c}+$ / $\mathrm{CD} 11 \mathrm{~b}+$ cells (marker for activated macrophages or dendritic cells), independent of body weight.

Despite the same amount of fat mass after the restriction period, HFD-CR mice did not only display a stronger reduction in macrophage infiltration, but also had a more prominent regulation of mitochondrial biogenesis, lipid synthesis, and, especially, carbohydrate metabolism. HFD$\mathrm{CR}$ did not result in a change in intake of a specific macronutrient, but rather reduced overall macronutrient intake, resulting in a lower energy intake. The change to LFD on the other hand changed macronutrient intake and concomitantly lowered energy intake. While carbohydrate intake was higher in the LFD than in HFD-CR (and in HFD for that matter), fat intake was lower (Fig. 2). These data strongly suggest that in particular the lowering of energy intake, and not so much the lowering of fat intake, is of primary importance to obtain beneficial metabolic effects of a diet intervention. Our results, however, contrast with previous studies showing that when HFD is restricted to the caloric intake of LFD fed mice, insulin resistance and hepatic steatosis are not prevented (de Meijer et al. 2010; Petro et al. 2004). The lack of more pronounced effects of the LFD may be due to a stronger decrease in energy intake during HFD restriction compared to the LFD (even though fat intake was higher in HFD-CR).

Alternatively, differences between the previous studies and our study may be explained by the use of different C57BL/6J sub-strains, with (our study) or without a functional Nnt gene. Although all C57BL/6J mice are known for their susceptibility to DIO (Nicholson et al. 2010), differences in macronutrient handling are likely to exist between different sub-strains in view of the role of NNT in, for example, the TCA cycle (Gameiro et al. 2013) and insulin release from pancreatic beta-cells (Freeman et al. 2006). 
A major difference in feeding regimen is whether the animals eat ad libitum (LFD mice) or whether they receive their food as a restricted single portion (HFD-CR mice). Feed was provided to the HFD-CR mice once a day prior to the dark phase. The mice consumed their portion very rapidly and were subsequently "fasted" until the next feeding time. This is supported by indirect calorimetry data showing that restricted mice shift to a cyclic pattern in which the initial phase shows increased fatty acid synthesis (RER > 1.1), followed by prolonged fatty acid oxidation $(\mathrm{RER}=0.7)$ as observed by ourselves (Fig. 3) and others (Bruss et al. 2010). The emergence of this cyclic pattern by time-restricted feeding has recently been proposed as a non-pharmacological intervention that could prevent obesity and its associated diseases (Hatori et al. 2012).

Another possibility is that the rate of restriction or the difference in protein intake is responsible for the differences found between LFD and HFD-CR. In a recent study on rats, it was shown that energy restriction, but not diet composition and protein level, impacted weight loss and adiposity (Chevalier et al. 2013). In humans, ad libitum access to food with a variety in the macronutrient composition promotes dietary adherence during weight loss and maintenance (Abete et al. 2010). Energy restriction, however, has shown consistently to produce weight loss and beneficial health effects. Extrapolation of our results on mice to the human situation remains difficult as we do not really mimic free-living conditions in this experiment (i.e., only one sort of diet is available).

The long fasting period due to the feeding regimen is further substantiated by decreased serum levels of FFA (Fig. 4e), which have shown to be correlated to the rate of lipolysis and fatty acid flux in adipose tissue (Duncan et al. 2007). Altogether, these findings suggest that food restriction forces the animals into a highly dynamic metabolic pattern, which may contribute to increased metabolic flexibility, possibly contributing to the enhanced health effects in the HFD-CR regimen.

The expression profile of eWAT showed enhanced mitochondrial carbohydrate metabolism as well as increased lipogenesis (Fasn) in HFD-CR (Fig. 7). It is striking that the two intervention groups are not regulated in a similar way, even though LFD mice are in the same physiological state as HFD-CR mice. Our molecular profiles showed no effects on mitochondrial fatty acid beta-oxidation as carnitine palmitoyl transferase transcripts were not regulated. But we do see an increase in Fasn regulation, indicative of fatty acid biosynthesis. Together with the increase in carbohydrate metabolism, this may suggest a shift to metabolism of carbohydrates, possibly to prevent energy depletion from WAT. Alternatively, it may be associated with a higher turnover of triglycerides in WAT as has also been observed after longterm HFD-CR (Duivenvoorde et al. 2011).
PPARGC1A is considered to be the master regulator of mitochondrial biogenesis. A strong body of evidence shows that increased expression of Ppargcla is associated with increased mitochondrial density in response to calorie restriction (Duivenvoorde et al. 2011; Higami et al. 2004; Linford et al. 2007). Here, we show a strong up-regulation of Ppargcl $\alpha$ as a response to HFD-CR. However, we do not see this reflected in levels of mitochondria as measured by ratio of mitochondrial to nuclear DNA nor by $\mathrm{CS}$ activity. This could indicate that PPARGC1A is stimulated via a different regulation pathway. Alternatively, the absence of an increased mitochondrial density may be due to the time frame of the current study, and it may be speculated that increased mitochondrial density will follow changes in Ppargcla and will become evident only after long-term HFD-CR. Although most papers show a strong correlation between measurements of mitochondrial density in WAT, one recent study reported a lack of association between several measured mitochondrial parameters (Cyt c, COXIV, Core 1, ATPS, and Ppargcla) in several organs, including WAT upon (LFD) CR in Wistar rats (Hancock et al. 2011). To be able to interpret markers of mitochondrial density, it is important to understand the relation between these markers in time.

In summary, using a mouse model of DIO, we demonstrated differential effects of LFD and HFD-CR weight loss strategies on inflammatory status of eWAT and on molecular mitochondrial carbohydrate and lipid metabolism of eWAT. From a physiological point of view, it seems that there is little difference in response between LFD and HFD-CR as both interventions mediate a more healthy profile. However, with a focus on molecular regulation, HFD-CR showed a more evident response by increasing both fat and carbohydrate metabolism, together with a more pronounced reduction in eWAT inflammation. Therefore, HFD-CR might be a more effective approach. This, however, needs to be confirmed in future experiments in mice and humans.

Acknowledgments We kindly acknowledge the extensive help of the members of human and animal physiology with tissue harvesting at the necropsy and everyone at the animal facility who was involved in this study. FH was supported by funding from graduate school WIAS (Wageningen Institute for Animal Science).

Conflict of interest The authors have declared no conflict of interests.

\section{References}

Abete I, Astrup A, Martinez JA, Thorsdottir I, Zulet MA (2010) Obesity and the metabolic syndrome: role of different dietary macronutrient distribution patterns and specific nutritional 
components on weight loss and maintenance. Nutr Rev 68:214-231

Astrup A, Toubro S, Raben A, Skov AR (1997) The role of low-fat diets and fat substitutes in body weight management: what have we learned from clinical studies? J Am Diet Assoc 97:S82-S87

Bruss MD, Khambatta CF, Ruby MA, Aggarwal I, Hellerstein MK (2010) Calorie restriction increases fatty acid synthesis and whole body fat oxidation rates. Am J Physiol Endocrinol Metab 298:E108-E116

Burton BT, Foster WR, Hirsch J, Van Itallie TB (1985) Health implications of obesity: an NIH consensus development conference. Int J Obes 9:155-170

Chevalier L, Bos C, Azzout-Marniche D, Fromentin G, Mosoni L, Hafnaoui N, Piedcoq J, Tome D, Gaudichon C (2013) Energy restriction only slightly influences protein metabolism in obese rats, whatever the level of protein and its source in the diet. Int J Obes (Lond) 37:263-271

Cinti S, Mitchell G, Barbatelli G, Murano I, Ceresi E, Faloia E, Wang S, Fortier M, Greenberg AS, Obin MS (2005) Adipocyte death defines macrophage localization and function in adipose tissue of obese mice and humans. J Lipid Res 46(11):2347-2355

de Meijer VE, Le HD, Meisel JA, Akhavan Sharif MR, Pan A, Nose V, Puder M (2010) Dietary fat intake promotes the development of hepatic steatosis independently from excess caloric consumption in a murine model. Metabolism 59:1092-1105

Duivenvoorde LP, van Schothorst EM, Bunschoten A, Keijer J (2011) Dietary restriction of mice on a high-fat diet induces substrate efficiency and improves metabolic health. J Mol Endocrinol 47:81-97

Duncan RE, Ahmadian M, Jaworski K, Sarkadi-Nagy E, Sul HS (2007) Regulation of lipolysis in adipocytes. Annu Rev Nutr 27:79-101

Freeman HC, Hugill A, Dear NT, Ashcroft FM, Cox RD (2006) Deletion of nicotinamide nucleotide transhydrogenase: a new quantitive trait locus accounting for glucose intolerance in C57BL/6J mice. Diabetes 55:2153-2156

Gameiro PA, Laviolette LA, Kelleher JK, Iliopoulos O, Stephanopoulos G (2013) Cofactor balance by nicotinamide nucleotide transhydrogenase (NNT) coordinates reductive carboxylation and glucose catabolism in the tricarboxylic acid (TCA) cycle. J Biol Chem 288:12967-12977

Goldstein DJ (1992) Beneficial health effects of modest weight loss. Int J Obes Relat Metab Disord 16:397-415

Goossens GH (2008) The role of adipose tissue dysfunction in the pathogenesis of obesity-related insulin resistance. Physiol Behav 94:206-218

Hancock CR, Han DH, Higashida K, Kim SH, Holloszy JO (2011) Does calorie restriction induce mitochondrial biogenesis? A reevaluation. FASEB J 25:785-791

Hatori M, Vollmers C, Zarrinpar A, DiTacchio L, Bushong EA, Gill S, Leblanc M, Chaix A, Joens M, Fitzpatrick JA et al (2012) Time-restricted feeding without reducing caloric intake prevents metabolic diseases in mice fed a high-fat diet. Cell Metab $15: 848-860$

Heilbronn LK, Noakes M, Clifton PM (1999) Effect of energy restriction, weight loss, and diet composition on plasma lipids and glucose in patients with type 2 diabetes. Diabetes Care 22:889-895

Herzig S, Raemy E, Montessuit S, Veuthey JL, Zamboni N, Westermann B, Kunji ER, Martinou JC (2012) Identification and functional expression of the mitochondrial pyruvate carrier. Science 337:93-96

Higami Y, Pugh TD, Page GP, Allison DB, Prolla TA, Weindruch R (2004) Adipose tissue energy metabolism: altered gene expression profile of mice subjected to long-term caloric restriction. FASEB J 18:415-417
Hoek-van den Hil EF, Beekmann K, Keijer J, Hollman PC, Rietjens IM, van Schothorst EM (2012) Interference of flavonoids with enzymatic assays for the determination of free fatty acid and triglyceride levels. Anal Bioanal Chem 402:1389-1392

Hoevenaars FP, Keijer J, Swarts HJ, Snaas-Alders S, BekkenkampGrovenstein M, van Schothorst EM (2013a) Effects of dietary history on energy metabolism and physiological parameters in C57BL/6J mice. Exp Physiol 98:1053-1062

Hoevenaars FPM, Bekkenkamp-Grovenstein M, Janssen RJRJ, Heil SG, Bunschoten A, Hoek-van den Hill EF, Snaas-Alders S, Teerds K, Van Schothorst EM, Keijer J (2013b) Thermoneutrality results in prominent diet induced body weight differences in C57BL/6j mice not paralleled by diet-induced metabolic differences. Mol Nutr Food Res. doi:10.1002/mnfr2013285

Kalupahana NS, Voy BH, Saxton AM, Moustaid-Moussa N (2011) Energy-restricted high-fat diets only partially improve markers of systemic and adipose tissue inflammation. Obesity (Silver Spring) 19:245-254

Kershaw EE, Flier JS (2004) Adipose tissue as an endocrine organ. J Clin Endocrinol Metab 89:2548-2556

Lagouge M, Argmann C, Gerhart-Hines Z, Meziane H, Lerin C, Daussin F, Messadeq N, Milne J, Lambert P, Elliott P et al (2006) Resveratrol improves mitochondrial function and protects against metabolic disease by activating SIRT1 and PGC-1alpha. Cell 127:1109-1122

Levy JC, Matthews DR, Hermans MP (1998) Correct homeostasis model assessment (HOMA) evaluation uses the computer program. Diabetes Care 21:2191-2192

Linford NJ, Beyer RP, Gollahon K, Krajcik RA, Malloy VL, Demas V, Burmer GC, Rabinovitch PS (2007) Transcriptional response to aging and caloric restriction in heart and adipose tissue. Aging Cell 6:673-688

Muurling M, Jong MC, Mensink RP, Hornstra G, Dahlmans VE, Pijl H, Voshol PJ, Havekes LM (2002) A low-fat diet has a higher potential than energy restriction to improve high-fat diet-induced insulin resistance in mice. Metabolism 51:695-701

Nicholson A, Reifsnyder PC, Malcolm RD, Lucas CA, MacGregor GR, Zhang W, Leiter EH (2010) Diet-induced obesity in two C57BL/6 substrains with intact or mutant nicotinamide nucleotide transhydrogenase (Nnt) gene. Obesity (Silver Spring) 18:1902-1905

Nisoli E, Tonello C, Cardile A, Cozzi V, Bracale R, Tedesco L, Falcone S, Valerio A, Cantoni O, Clementi E et al (2005) Calorie restriction promotes mitochondrial biogenesis by inducing the expression of eNOS. Science 310:314-317

Park JY, Wang PY, Matsumoto T, Sung HJ, Ma W, Choi JW, Anderson SA, Leary SC, Balaban RS, Kang JG, et al. (2009) p53 improves aerobic exercise capacity and augments skeletal muscle mitochondrial DNA content. Circ Res 105: 705-712, $711 \mathrm{p}$ following 712

Petro AE, Cotter J, Cooper DA, Peters JC, Surwit SJ, Surwit RS (2004) Fat, carbohydrate, and calories in the development of diabetes and obesity in the C57BL/6J mouse. Metabolism 53:454-457

Ronchi JA, Figueira TR, Ravagnani FG, Oliveira HC, Vercesi AE, Castilho RF (2013) A spontaneous mutation in the nicotinamide nucleotide transhydrogenase gene of C57BL/6J mice results in mitochondrial redox abnormalities. Free Radic Biol Med 63C:446-456

Scarpulla RC (2008) Transcriptional paradigms in mammalian mitochondrial biogenesis and function. Physiol Rev 88:611-638

Surwit RS, Kuhn CM, Cochrane C, McCubbin JA, Feinglos MN (1988) Diet-induced type II diabetes in C57BL/6J mice. Diabetes 37:1163-1167

Van Schothorst EM, Franssen-van Hal N, Schaap MM, Pennings J, Hoebee B, Keijer J (2005) Adipose gene expression patterns of 
weight gain suggest counteracting steroid hormone synthesis. Obes Res 13:1031-1041

Wang P, Mariman E, Renes J, Keijer J (2008) The secretory function of adipocytes in the physiology of white adipose tissue. J Cell Physiol 216:3-13

Wang Q, Perrard XD, Perrard JL, Mansoori A, Raya JL, Hoogeveen R, Smith CW, Ballantyne CM, Wu H (2011) Differential effect of weight loss with low-fat diet or high-fat diet restriction on inflammation in the liver and adipose tissue of mice with dietinduced obesity. Atherosclerosis 219:100-108

Zechner R, Kienesberger PC, Haemmerle G, Zimmermann R, Lass A (2009) Adipose triglyceride lipase and the lipolytic catabolism of cellular fat stores. J Lipid Res 50:3-21

Zhou YP, Grill VE (1994) Long-term exposure of rat pancreatic islets to fatty acids inhibits glucose-induced insulin secretion and biosynthesis through a glucose fatty acid cycle. J Clin Invest 93:870-876 